\title{
Design of Alcohol Content Detector Based on Single Chip Microcomputer
}

\author{
Fu Xiuwei ${ }^{1, a,{ }^{*},}, \mathrm{Fu} \mathrm{Li}^{2, \mathrm{~b}}$ and Wang Yifeng ${ }^{3, \mathrm{c}}$ \\ ${ }^{1}$ College of Information \& Control Engineering, Jilin Institute of Chemical Technology, Jilin 132022, \\ China \\ ${ }^{2}$ College of Information \& Control Engineering, Jilin Institute of Chemical Technology, Jilin 132022, \\ China \\ ${ }^{3}$ College of Information \& Control Engineering, Jilin Institute of Chemical Technology, Jilin 132022, \\ China \\ afxw7720268@163.com, bfuli247012412@126.com, c1637623359@qq.com \\ ${ }^{*}$ Corresponding author
}

Keywords: Alcohol content, Drunk driving, Microcontroller.

\begin{abstract}
An intelligent locking system for preventing drink driving is studied in this paper. This system is mainly determined by the alcohol sensor to detect whether the alcohol content exceeds the standard by the single-chip microcomputer control the ignition of the car can start. Because the system is installed in a car, traffic accidents caused by drunk driving can be effectively prevented.
\end{abstract}

\section{Introduction}

Drinking and driving is a social phenomenon that cannot be underestimated. The alcohol detection of the driver by the traffic police can only be carried out after the accident. Therefore, it is necessary to study a smart locking system for preventing drink driving. This article describes a microcontroller-based alcohol content detector. Mainly by the microcontroller to determine and control whether the car's engine allows ignition start.

\section{Project design}

In this system, the driver must actively exhale and allow the car to start after the test has not exceeded the limit. The system can be installed in the car dashboard or steering wheel position. When the driver inserts the key, the alcohol sensor detects the driver's breath. The detection signal is amplified, filtered, and then transmitted to the microcontroller after $\mathrm{A} / \mathrm{D}$ conversion is finished. The overall scheme design block diagram is shown in Fig.1.

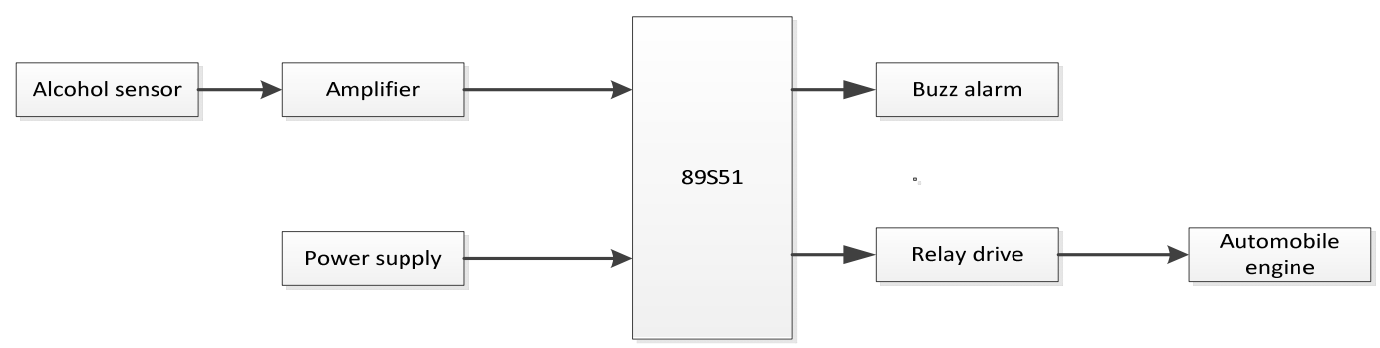

Fig. 1. Overall structure of the system

\section{Hardware circuit design}

The system hardware is mainly composed of a power module, an alcohol detection module, a core control module, an A/D conversion module, a relay drive module, and a display alarm module. 


\subsection{Power Module}

The system needs $+5 \mathrm{~V}$ voltage to supply power. Because the system is installed in the car, it uses $+12 \mathrm{~V}$ power and $+9 \mathrm{~V}$ in the car. The dry battery is converted to $+5 \mathrm{~V}$ by the voltage regulator module LM7805 to supply power to the system. Power module shown in Fig. 2.

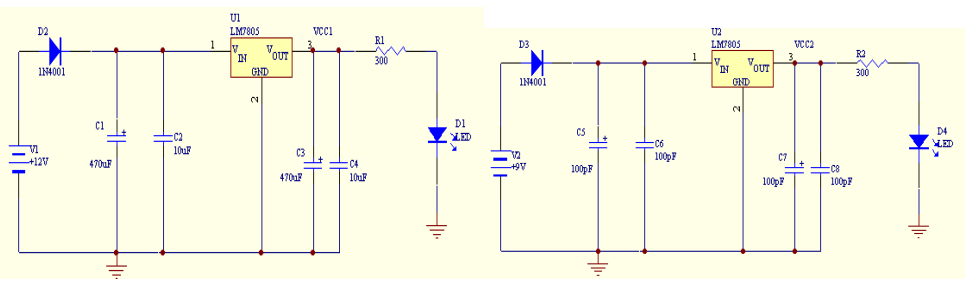

Fig. 2. Schematic diagram of the power module

\subsection{Core Control Module}

The core control module of this system is mainly composed of AT89S51 single-chip microcomputer, crystal oscillator circuit and reset circuit. This module mainly carries out the judgment on the signal sent after detecting the amplification and controls the ignition and alarm. The schematic diagram is shown in Fig. 3.

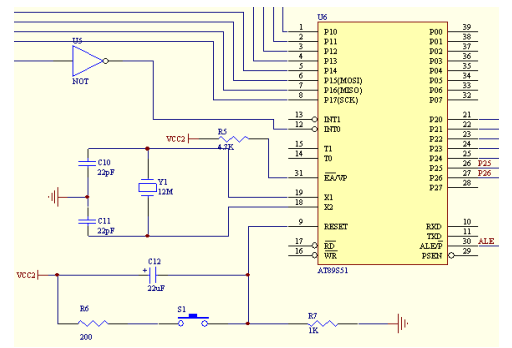

Fig. 3. Schematic diagram of the core control module

\subsection{Signal detection, amplification and conversion module}

This module is mainly composed of alcohol sensor QM-J3 alcohol sensor, operational amplifier AD632 and analog/digital converter ADC0809. When the QM-J3 element is exposed to ethanol vapor during exhalation, its electrical conductivity rapidly increases as the gas concentration increases and the voltage signal also increases. After the voltage signal is detected, the amplifier circuit, and the A/D conversion circuit are converted into digital quantities, they are sent to the SCM to determine the threshold and detect whether the alcohol content in the gas exhaled by the driver is excessive. The principle is shown in Fig. 4.

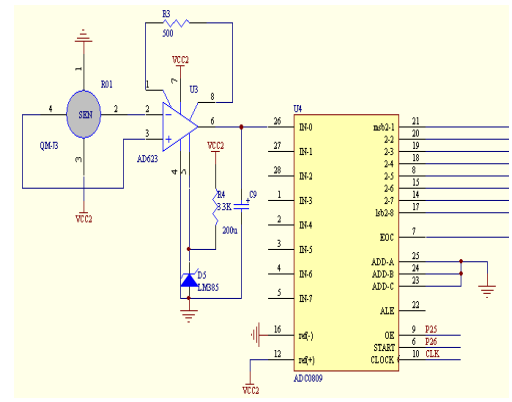

Fig. 4. signal detection, amplification, conversion circuit schematic

\subsection{Wireless Transmitter and Receiver Module}

When the MCU determines whether the alcohol concentration in the human body exceeds the standard, the system will drive the relay accordingly. If theconcentration is too high, the automobile 
engine power is cut off so that the car cannot be started, thereby effectively preventing drivers from drinking and driving. Relay uses double pole double throw relay HRS2H-S-DC5V, low power relay. The principle is shown in Fig. 5.

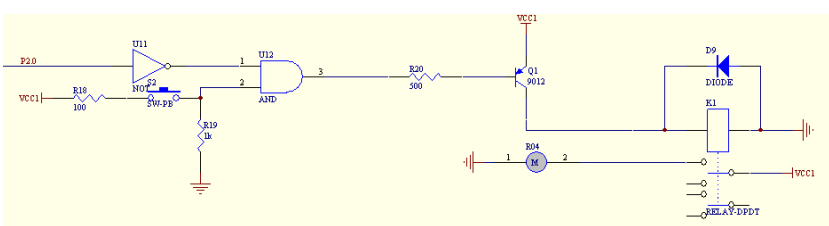

Fig. 5. Relay drive module schematic

\subsection{Relay Drive Module}

The acquisition of the signal adopts the mature F04B wireless transmission module, F04B is the sending module, or the LC oscillator, adopts SMT technology, resin encapsulation, special small size, extremely low power consumption, especially suitable for short-distance use of small products. The $\mathrm{J} 04 \mathrm{E}$ receiving module is principle of wireless transmitter and receiver module is shown in Fig. 6 .
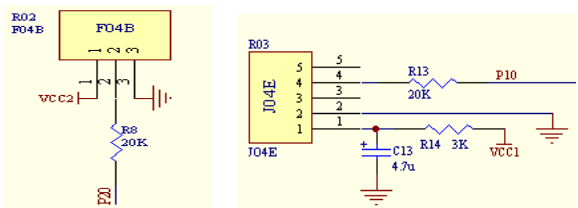

Fig. 6. System emission, receiving schematic

\subsection{Relay Drive Module}

The red light is the power indicator and is on during work; the green light is the breath indicator light, and the yellow light indicates that the breath is valid. The buzzer sounds when the detected fine concentration is exceeded. The driver is advised not to drink and drive. The principle is shown in Fig. 7.
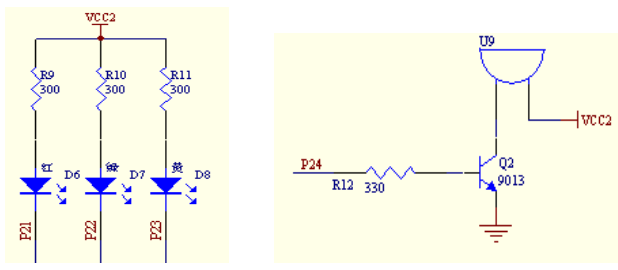

Fig. 7. The alarm module

\section{System software design}

The software part implements functions such as breath identification, alcohol content detection, and detection result action according to the system function settings. Due to the limitation of display, this article gives the main functions in the form of a flow chart. The main program flow is shown in Fig. 8. 


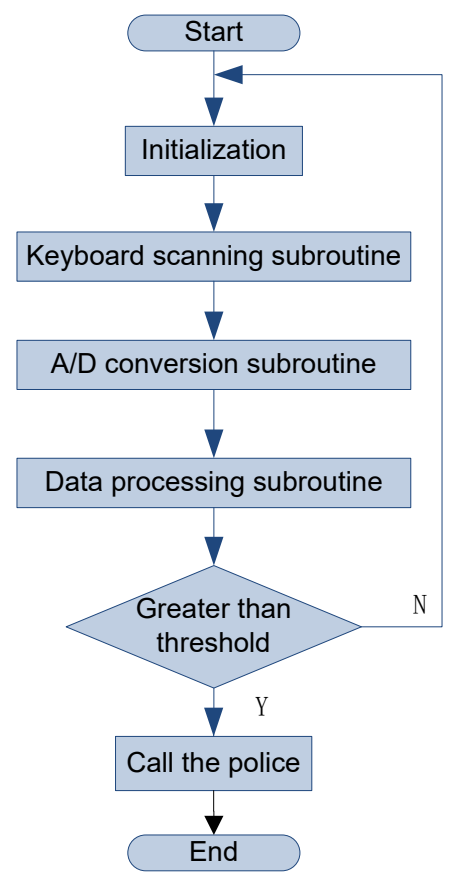

Fig. 8. system main program flow chart

\section{Conclusion}

The microcomputer-based alcohol content detector is designed to prevent traffic accidents. This article describes the entire design through the description of hardware components and software workflows. Because this system is small, inexpensive, easy to use, and it is installed on the car, it is not easy to uninstall, so it has good effect on preventing drink driving. It has good application value in practical applications.

\section{References}

[1] Li Qiang. 51 Series Microcontroller Application Software Programming Technology[M]. Beijing: Beijing University of Aeronautics and Astronautics Press, 2009.

[2] Li Jianzhong. Principles and Applications of Microcontroller[M]. Xi'an: Xidian University Press, 2002.

[3] Hu Xiangdong. Sensor and Detection Technology[M]. Beijing: Mechanical Industry Press, 2007. 